LIVER CANCER

\section{Platelets with dangerous cargo}

Platelets are involved in

pathophysiological conditions

associated with metabolic disease, including obesity, atherosclerosis and liver disease, but it is unclear how this occurs mechanistically. Malehmir et al. show that platelet recruitment to the liver contributes to development of nonalcoholic steatohepatitis (NASH) and hepatocellular carcinoma (HCC) through platelet glycoprotein Iba (GPIba).

The authors analysed platelets in the livers of mice fed a cholinedeficient high-fat diet (CD-HFD), which induces NASH around 6 months after the diet is started. Compared with normal chow diet (NCD)-fed controls, livers from CD-HFD mice had more platelets, which aggregated into larger clumps. Increased intrahepatic platelet numbers were also found in four other dietary and genetic mouse NASH models, as well as in patients with $\mathrm{NASH}$, compared with healthy controls. However, in mice fed a lower calorie HFD (leading to steatosis and/or insulin resistance), intrahepatic platelet numbers remained the same as in controls, thereby linking increased intrahepatic platelet numbers specifically to NASH.

Aspirin-clopidogrel (Asp-Clo) is an antiplatelet therapy (APT) that blocks platelet activation and aggregation. This treatment reversed increased platelet numbers and aggregation in CD-HFD-fed mice but did not affect the higher weight gain or body fat observed in CD-HFD-fed mice than in NCD-fed mice. However, Asp-Clo improved glucose tolerance, reduced liver triglycerides and improved cholesterol levels in CD-HFD-fed mice compared with untreated CD-HFD-fed mice. This led to reduced deposition of fat and lobular inflammation, overall preventing the development of NASH.

NASH development is accompanied by immune cell infiltration in the livers of mice. Of note, Asp-Clo reduced intrahepatic infiltration of effector $\mathrm{CD}^{+} \mathrm{T}$ cells and natural killer cells and activation

of inflammatory carcinogenic signalling pathways including nuclear factor- $\kappa \mathrm{B}$ and p38 MAPK. In addition, Kupffer cell activation was reduced. Importantly, CD-HFD-fed mice developed overt tumours in their livers by 12 months on this diet, which could be reduced by Asp-Clo treatment. Tumours were not detected in livers of NCD-fed mice.

Prior to NASH, mice fed a CD-HFD show symptoms of simple steatosis (also known as nonalcoholic fatty liver (NAFL)), which can develop in the first two months of starting the diet. Intravital microscopy of mice during the first 8 weeks of CF-HFD or NCD showed platelets increasingly accumulating and aggregating in the livers of CF-HFD-fed mice from 4 to 8 weeks but not in NCD-fed mice. 3D image reconstruction showed that platelets interacted with Kupffer cells. Although no other infiltrating cell types were detected, mild steatosis and hepatocyte swelling occurred as early as 4 weeks after starting the diets.

Were Kupffer cells involved in early platelet recruitment? To explore this question, the researchers inhibited a potential interaction between these cells by depleting Kupffer cells using clodronate liposomes (CLL) and/or blocking the extracellular matrix molecule hyaluronan, which platelets interact with via the cell surface receptor CD44, using hyaluronidase (HYAL). Indeed, CLL

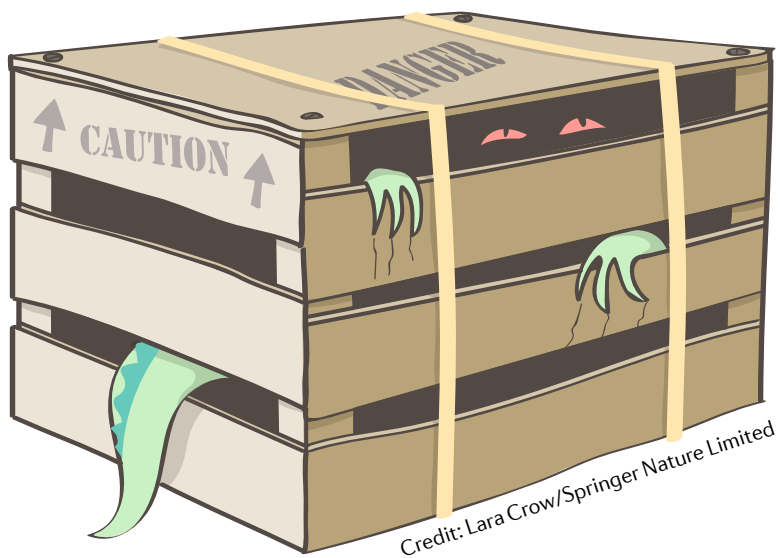

alone or together with HYAL in CD-HFD-fed mice with borderline NASH reduced Kupffer cell numbers, and CLL and/or HYAL reduced intrahepatic platelet accumulation. This was accompanied by reduced nonalcoholic fatty liver disease (NAFLD) activity score (NAS, measuring steatosis, lobular inflammation and liver cell injury) and liver damage (the latter only in response to HYAL and not CLL). In addition, antibody-mediated blockade of the interaction between CD44 and hyaluronan significantly reduced NAS and liver damage.

The researchers then investigated whether specific platelet cargo was mediating liver damage. Experiments involving several genetically engineered mouse models lacking either $\alpha$-granules as platelet cargo, the ability of platelets to aggregate or vascular adhesion molecules and their ligands showed that platelet recruitment and cargo were contributing to NASH, whereas platelet aggregation was not. When platelet-derived GPIba, which contributes to platelet attachment and activation, was blocked by a specific antibody in mice fed with CD-HFD, intrahepatic platelet accumulation was reduced, along with NAS, liver damage and immune cell infiltration. However, this treatment did not prevent NASH development in the earlier stages, possibly owing to a lack of unidentified GPIba ligands. In $G P I b \alpha^{-/-}$mice expressing a fusion protein of GPIba in which the ligand binding domain was replaced by the a-subunit of the human interleukin-4 receptor, a CD-HFD did not induce changes associated with NAFL or NASH. These mice also had significantly lower fibrosis upon longterm treatment of up to 12 months than did controls and did not develop HCC.

APT and anti-GPIba seem to be promising therapeutic modalities for NASH and NASH-induced HCC. However, appropriate timing in the development of NASH and potential combinations need to be tested in further preclinical studies.

Ulrike Harjes

ORIGINAL ARTICLE Malehmir, M. et al. Platelet GPIba is a mediator and potential interventional target for NASH and subsequent liver cancer.

Nat.Med. 25, 641-655 (2019) 